\title{
Integration of Laser-support Fiber Adjustment in Opto-electronic Modules
}

\author{
J.H.C. van Zantvoort, S.G.L. Plukker, E.C.A. Dekkers, G.D. Khoe, A.M.J. Koonen and H. de Waardt \\ COBRA Research Institute, Eindhoven University of Technology \\ 5600 MB Eindhoven, The Netherlands \\ j.h.c.v.zantvoort@tue.nl
}

\begin{abstract}
In this study, practical assembly methods are developed to connect fibers to photonic chips. In contrast to trial and error efforts using laser hammering or mechanical bending approaches, we introduce the integration of laser-assisted mechanical micro-scale deformation within the module itself. The technology is based on introducing a locally compressive plastic strain into the fiber supports. We can then obtain a predictably adequate correction of the pre-aligned fibers, a necessary step because during the fixation process degradation of the aligned positions can occur. We manufacture modules for single fiber configurations and more advanced modules for fiber-array configurations. We have also started numerical simulations of laser-adjusting to investigate further design aspects.
\end{abstract}

\section{Introduction}

The alignment requirements for lensed fiber tips related to planar waveguides are very tight, typically in the submicrometer range. This requirement can be met using commercially available manipulation systems. However, fixation of the optimum position is a much greater challenge. This is because two main problems can cause degradation of aligned positions:

1) When laser welding is used to fix the aligned positions, the metal parts fuse together upon solidification to form a weld joint. However, shrinkage forces develop during this solidification. Consequently, the shrinkage forces produce misalignment, known as the post-weldshift (PWS).

2) When the tools necessary for the alignment of the components are released, the internal stresses between the aligned components are relieved, resulting in deviation of the positions. We will refer to this as the post-release-shift (PRS).

During the last decade, different kinds of fiber-clip designs were invented for applications in butterfly-type packages [1]. In all designs, both PWS and PRS are compensated for as much as possible by using laserhammering techniques. With this technique, re-adjusting already welded parts is executed by repeatedly re-welding the parts using asymmetric laser pulses. Another strategy is to deform the welded parts using mechanical forces in order to bend the components into the optimum position. However, the yield produced using these methods is unknown.

Within the COBRA Research Institute, we investigate alternatives for the accurate pigtailing of optical chips in order to implement new prototype devices in system- related areas. Our preference is to use laser contraction techniques that allow for a well-controlled step-by-step alignment approach across a broad range of fiber alignment applications.

\section{Laser adjust process}

The alignment of the fiber tips is based on introducing a locally compressive plastic strain in the material of the fiber-supports. The local strain is generated by irradiating certain parts of the fiber-support at the lower output energies of a Nd:YAG laser welder. Masubuchi [2] describes in detail the transient thermal stresses of a beadon-plate weld, produced by the movement of a welding arc. Similar stresses are produced when a steady laser beam produces a circular and local weld pool.

In brief, assume that a metal sheet, as sketched in figure 1 (a), is part of a fiber-support. Both in-plane stress distributions $\left(\sigma_{\mathrm{x}}\right)$ and $\left(\sigma_{\mathrm{y}}\right)$ are equal to $(\sigma)$ and the initial stress in the sheet is considered to be zero. The tensile and compressive stresses are defined as presented in Fig. 1 (a). In Fig. 1 (b), a laser pulse is illustrated that heats up the material locally and causes the local temperature to reach the melting point of the material. The molten metal will not support a load and therefore the thermal stresses in the center of the molted metal are close to zero. The surrounding non-heated material obstructs the thermal expansion of the heated area, whereas the yield strength decreases rapidly above $300{ }^{\circ} \mathrm{C}$. This results in a situation where the thermal stresses are as high as the yield stresses at the corresponding temperature profiles, and are symmetrical around the center of the heated area. Consequently, plastic deformation of material occurs and the stresses generated in this region become compressive. The compressive stresses are in balance with tensile stresses in the regions further away from the heated center, as illustrated in Fig. 1 (b). After the heated part of the material has cooled down to room temperature, contraction arises from the plastic deformation, generating residual tensile stresses in regions in the heated area of the metal sheet [Fig. 1 (c)]. The residual tensile stress then generates a locally compressive plastic strain.

To investigate this thermal-mechanical interaction we simulate laser adjustment using the commercially available software MSC. Marc Mentat. A thin metal sheet with dimensions of $10 \times 1 \times 0.25 \mathrm{~mm}$ is shown in Fig. 2 (a). Due to symmetry conditions, only one-half of the spot heating is analyzed using the weld fill option. Both the weld filler and the base material of the sheet are of the same steel material. The temperature dependencies of mechanical properties, like Young's modulus, the coefficient of thermal expansion, yielding, and the 
thermal properties of specific heat and conductivity are considered here. Due to the stirring effect of the molten metal, the thermal conductivity is increased to a high value of $120 \mathrm{~W} / \mathrm{m}^{2} \mathrm{~K}$ beyond the melt temperature [3], [4], [5]. Solid-liquid transition is accounted for by providing a latent heat of fusion of $250 \mathrm{~kJ} / \mathrm{kg}$ with a solidus temperature of $1370{ }^{\circ} \mathrm{C}$ and a liquidus temperature of $1400{ }^{\circ} \mathrm{C}$. A convection film boundary condition is applied to all edges of the sheet as well as to heat transfer to the surroundings, caused by radiation. The sheet is fixed in the $x-, y-$, and $z$-directions at the edge $A$, and over the length $\mathrm{L}$ in the $\mathrm{y}$-, and z-directions [see Fig. 2 (a)]. The initial temperature is $30^{\circ} \mathrm{C}$ and at $\mathrm{T}=100 \mathrm{~ms}$, a volumetric weld flux pulse with a pulse duration of $5 \mathrm{~ms}$ and an energy of $2 \mathrm{~J}$ is applied to the weld filler as a boundary condition. The weld radius was taken as 0.5 $\mathrm{mm}$ and the efficiency of the energy transfer between the laser pulse and the sheet was taken to be $70 \%$ [5]. The calculated temperature distribution, which is generated directly after the laser pulse, is shown in Fig 2 (a). The temperature and corresponding stress distribution calculated over the length $\mathrm{L}$ of the metal sheet is shown in Fig. 2 (c). The residual tensile stress distribution after the heated part has cooled down to room temperature is shown in Fig. 2 (d). As a result, the calculated displacement of node 1519 [denoted in Fig. 2 (a)], as a function of time, is given in Fig 2 (b). During the laser pulse duration of $5 \mathrm{~ms}$, the expansion in the metal sheet of $5.2 \mu \mathrm{m}$ is calculated. Following this, after cooling down to room temperature, the net displacement from the initial position is $2.6 \mu \mathrm{m}$. This basic model of laser adjusting will be further implemented in advanced design models in order to explore aspects of design related to optoelectronic modules.

An example of a practical laser-adjust experiment is shown in Fig. 3. The displacement of a certain position of a metal sheet is measured with a sample frequency of 10 $\mathrm{kHz}$. After activating the Nd:YAG laser welder at $\mathrm{T}=0.5$ $\mathrm{s}$, an expansion of $5 \mu \mathrm{m}$ is measured during the $5 \mathrm{~ms}$ laser pulse. The net displacement $\Delta \mathrm{L}$ in this experiment is 3 $\mu \mathrm{m}$. The level of laser energy necessary for plastic deformation in the concerned sheet is approximately 1.5 $\mathrm{J}$. The displacement achieved by one laser pulse is on the order of $0.1 \mu \mathrm{m}-5 \mu \mathrm{m}$, which is achieved by adjusting the laser energy to between $1.5 \mathrm{~J}$ and $4.5 \mathrm{~J}$.

\section{Applications of laser adjustment in micro-modules}

To apply this technology, metal sheets or tuning frames must be implemented in micro-systems so that manipulation of the fibers can be executed. An overview is given in the next Section for single fiber and fiber-array configurations.

\section{Single fiber configurations}

An example of a mechanical micro-system designed for one fiber is shown in Fig. 4 (a) and consists of three elements: (1) a fiber ferrule including the fiber, (2) a solid sub-support and (3) two tuning frames, already mounted at an angle of $90^{\circ}$ relative to each other and to the fiber ferrule. The combination of the fiber ferrule (1) and tuning frames (3) is aligned using a pneumatic pair of tweezers and subsequently welded to the solid subsupport (2) at positions (P) and ( $\left.\mathrm{P}^{\prime}\right)$, where the back part position ( $\left.\mathrm{P}^{\prime}\right)$ is not visible in Fig 4 (a). The two mounting positions, $\mathrm{P}$ and $\mathrm{P}$ ', act as pivot points for the fiber ferrule. This allows for the realization of a predictable two-dimensional fine-tune mechanism of the fiber tip using laser adjusting on both tuning frames (3). The definition of the coordinate system and the displacement of the fiber tip as a function of a certain heated position at the tuning frames are given in Fig. 4 (b).

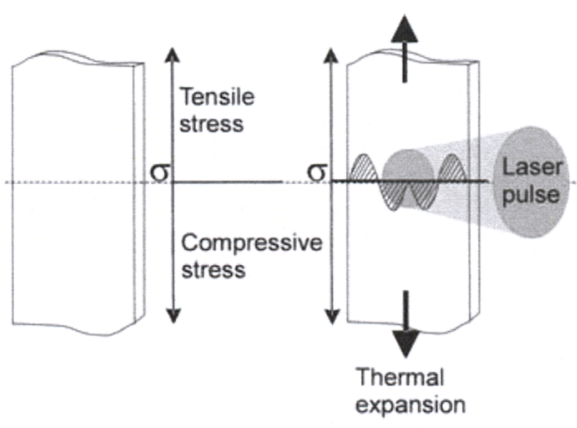

(a)

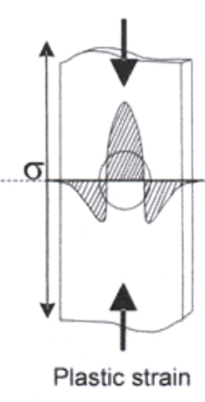

(c)

Fig. 1. Principle of laser-support adjustment: (a) the initial stress ( $\square$ ) in a metal sheet is assumed to be zero. (b) Heating up the metal beam locally at lower output energies of a Nd:YAG laser welder. Thermal expansion occurs, resulting in plastic deformation. The center of the irradiated part reaches the melting point temperature and the stress in this region becomes compressive. (c) Contraction arises from the plastic deformation of the metal sheet after the heated zone has been cooled to room temperature. The nature of the stress in the center is tensile generating plastic strain. The plastic strain is used for the mechanical adjustment of optical fibers
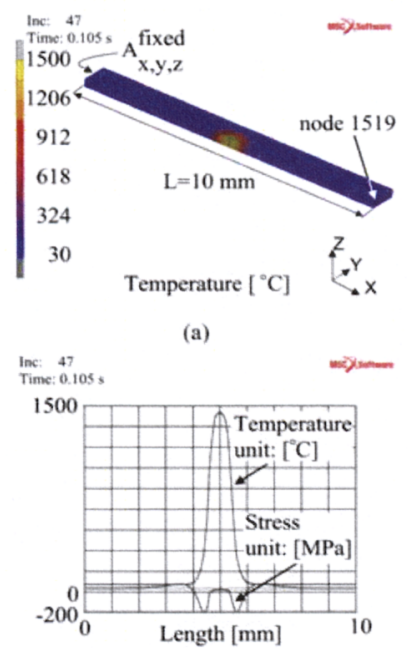

(c)

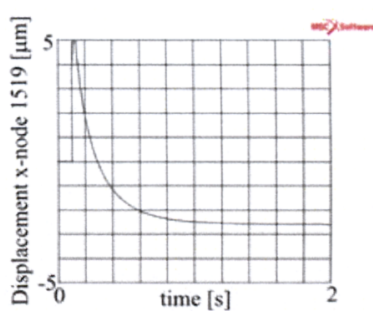

(b)

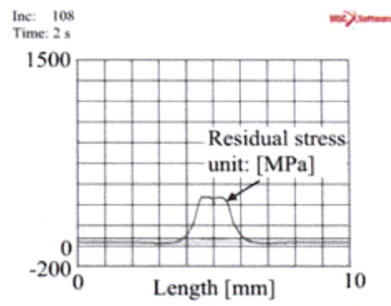

(d)

Fig. 2. Simulation results of laser-support adjustment. (a) Temperature distribution of a $3 \mathrm{D}$ model of a metal sheet $(10 \times 1 \times 0.25 \mathrm{~mm})$ directly after the laser pulse with an energy of $2 \mathrm{~J}$. (b) X-position of node 1519 [Fig 2 (a)] as a function of time. (c) Plot of the temperature and compressive stress distribution calculated over the length $\mathrm{L}$, directly after the laser pulse, and (d) residual tensile stress distribution over the same length $\mathrm{L}$ after $2 \mathrm{~s}$. 


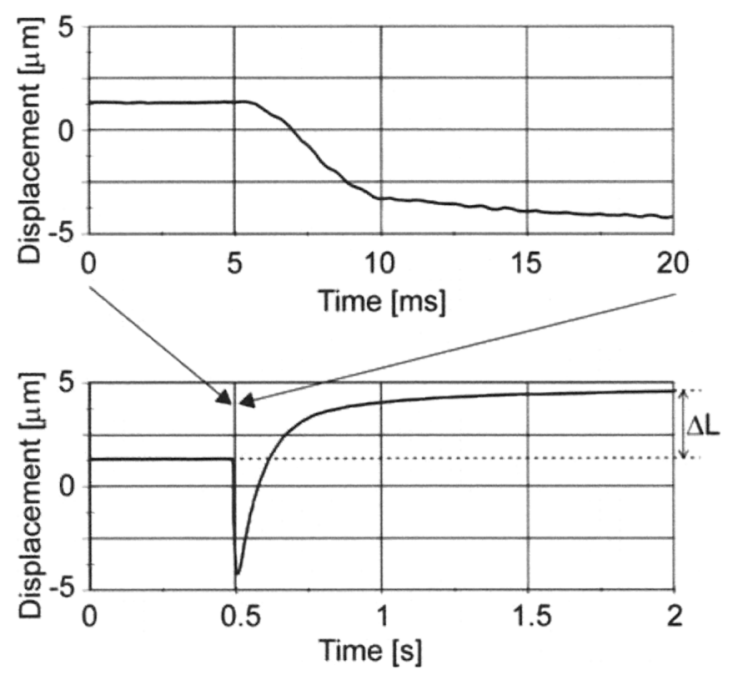

Fig. 3. Experimentally measured dynamic response of laser-adjustment. The displacement is measured at a certain position below the irradiated center of a metal sheet, which is sketched in Fig. 1. Upper graph: first expansion of $5 \mu \mathrm{m}$ is measured during the laser pulse duration of $5 \mathrm{~ms}$. After the cooling down period (lower graph), shrinkage in the metal sheet is measured, resulting in a net displacement $\Delta \mathrm{L}$ of $3 \mu \mathrm{m}$.

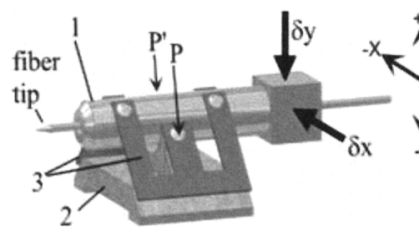

(a)

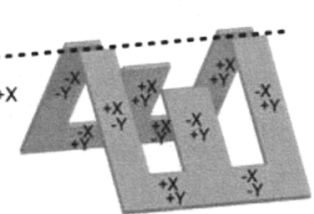

(b)
Fig. 4 (a). Schematic presentation of a single fiber support micro-system. (1) fiber ferrule including fiber, (2) solid sub-support, and (3) two tuning frames already mounted to the fiber ferrule (1). The fiber ferrule and tuning frames combination is aligned using pneumatic tweezers and permanently welded at positions $\mathrm{P}$ and $\mathrm{P}$ '. These positions act as a pivot point, using laser-adjustment to create a predictable two-dimensional fine-tune mechanism. (b) The displacement of the fiber tip in the $+X,-X$, $+Y$, and $-Y$ direction as function of the laser-adjusted positions executed at the tuning frames.

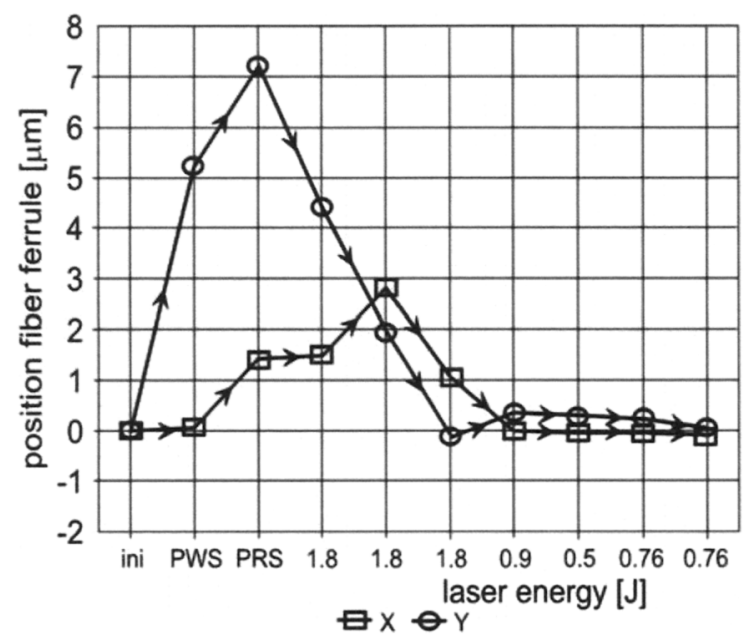

Fig. 5. Pigtail process executed at a prototype micro-system. The shifts of $7.2 \mu \mathrm{m}$ (X-direction) and $1.5 \mu \mathrm{m}$ (Y-direction) as a result of PWS and PRS are fully compensated for using laser-adjustment.
Dummy modules are fabricated to investigate the PWS and PRS, the range of manipulation, the step-size, and to verify the direction of fiber ferrule manipulation as a function of the heated position at the tuning frames of the micro-system. The magnitude of the PWS is on the order of $5 \mu \mathrm{m}$, while the contribution of the PRS is somewhat lower. The range that can be adjusted is approximately $20 \mu \mathrm{m}$ and is sufficient to compensate for the PWS and PRS. By reducing the laser energy, step sizes of $0.1 \mu \mathrm{m}$ are measured. In general, we observed the trend as described in Fig 4 (b). The position of the fiber ferrule is measured continuously in the linear $\delta \mathrm{x}$ and $\delta \mathrm{y}$ directions, located at the opposite position of the fiber tip as shown in Fig. 4 (a). An example of a laser-adjustment process is visualized in Fig 5. The shift of the fiber ferrule caused by PWS is $0.1 \mu \mathrm{m}$ and $5.2 \mu \mathrm{m}$ in the lateral Xand transverse Y-direction, respectively. After disconnecting the pneumatic tweezers, we measured an additional shift of $1.4 \mu \mathrm{m}$ and $2 \mu \mathrm{m}$ in the X- and Ydirections. After seven laser shot adjustments, the fiber ferrule was manipulated back to the initial position. At the moment of writing this paper, we are working towards the integration of this principle with two fiber connections, which are oriented opposite to each other in order to pigtail quantum-dot based semi-conductor optical amplifiers [6].

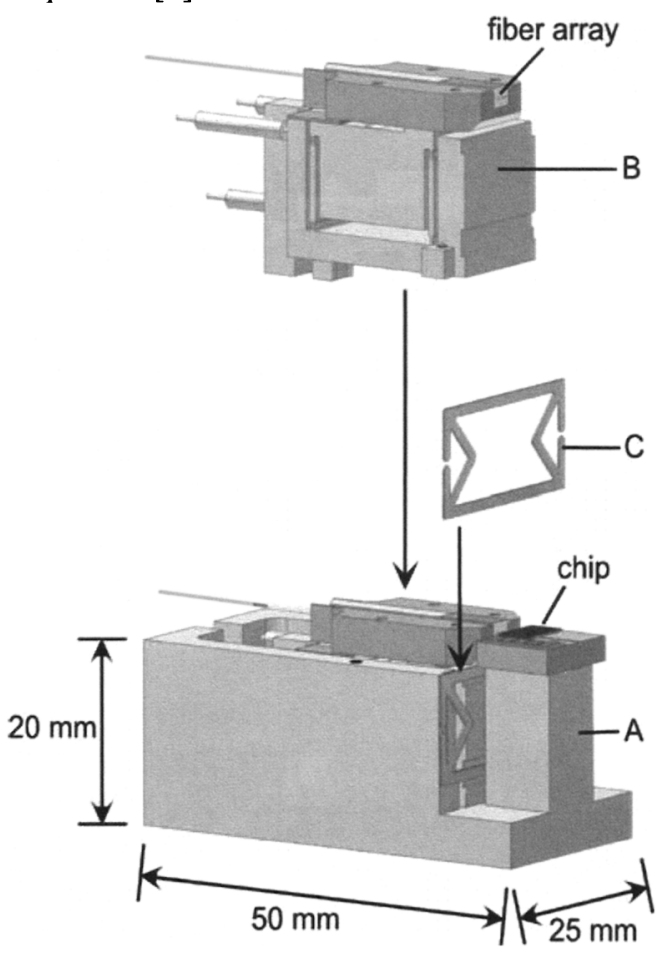

Fig. 6. Schematic presentation of an assembly designed for fiber-arrayto-photonic-chip pigtailing including laser-adjustment. Top: inner adjustable actuator module (B) which is mounted in the "U-shaped" module (A) and after aligning is permanently fixed with the tuning frame (C). Module (B) consists of a parallelogram to enable linear movement of the fiber-array toward the chip facet. The other two linear, and one rotational, degrees of freedom are possible using three elastic pins, which are connected between module (A) and module (B). Any possible misalignments during the assembly procedure can be compensated for using laser-assisted adjustment of the inner actuator module (B) to the initial optimum position by introducing local strain in the tuning frame $(C)$. 


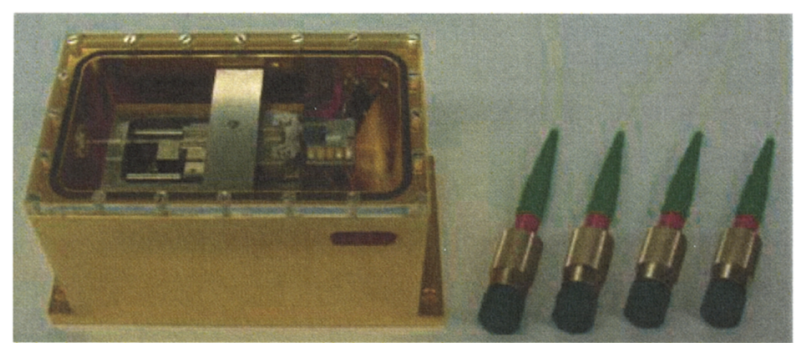

Fig. 7. Photograph of a realized device for fiber array configuration. Dimensions $7.5 \mathrm{~cm}$ (l) $\times 4.5 \mathrm{~cm}$ (b) $\times 4 \mathrm{~cm}$ (h).

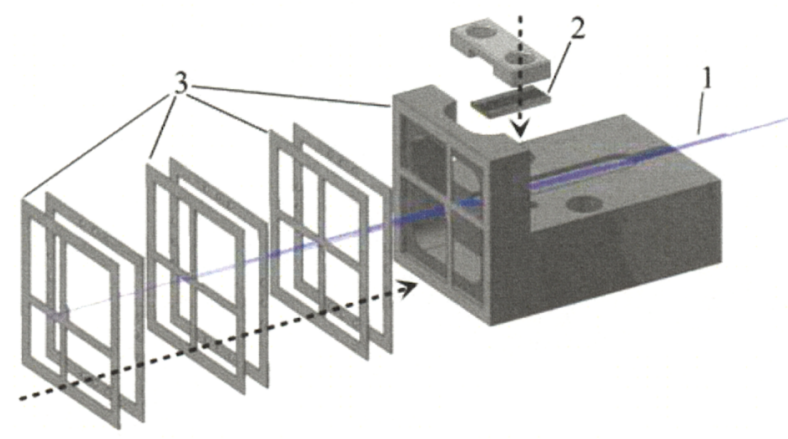

Fig. 8. Design to align fibers individually in a fiber-array. The fibers (1) are mounted in a V-groove substrate (2) and the fiber ends are connected in separated tuning frames (3). These tuning frames enable positioning of the fiber tips using laser-adjustment.

\section{Array configurations}

Laser adjustment integration for fiber-array applications is realized with the assembly presented in Fig. 6. The chip is mounted on module (A) and the fiberarray is mounted on the inner adjustable actuator module (B). With the tuning frame (C), it is possible to fix module (B) and to compensate for any PWS and PRS. Module (B) consists of a parallelogram to enable linear translation of the fiber-array towards the chip facet. The other two linear translations and one rotational degree of freedom are realized using three elastic pins, which are connected between modules (A) and (B). The position of the inner actuator module (B) can be adjusted using three temporarily connected piezo-electric actuators with active feedback. The position of module (B) relative to module (A) is also measured continuously using three noncontact displacement sensors based on inductive technology. Different types of photonic integrated circuits are pigtailed with this method. Successful alignment compensation on the order of $0.1 \mu \mathrm{m}-3 \mu \mathrm{m}$ is then established. A photograph of a complete packaged optoelectronic module is shown in Fig. 7. Detailed information on the complete assembly procedure can be found in ref. [7].

The maximum coupling efficiency between the lensed fiber tips of a fiber-array and waveguides is an average coupling loss, which results from a trade-off among the inaccuracies of the fiber-array, which result from the lenseccentricity of each individual fiber tip and the accuracy of the V-groove support. The average coupling loss is -6 $\mathrm{dB} /$ fiber tip-waveguide. To overcome the problem of core-eccentricity, we used laser-adjustment to assemble fiber-arrays whereby each individual fiber can be aligned independently. The design is shown in Fig. 8. The fibers (1) are mounted in a V-groove substrate (2) and the fiber ends are mounted in separated adjust frames (3). The accuracy of the fiber tip positions of the pre-assembled fiber-array was measured to be $\pm 8 \mu \mathrm{m}$. After the laseradjust process, the accuracy of the fiber-array is \pm 0.25 $\mu \mathrm{m}$. Detailed information on this can be found in ref. [8]. The coupling losses of the lensed fiber tips and InP-based waveguides are improved from $-6 \mathrm{~dB}$ to $-3 \mathrm{~dB}$. The laseradjusted, high precision arrays are mounted on commercially available 6-axis manipulation systems and their use has been demonstrated for multi-port photonic chip characterization within the COBRA Research Institute.

\section{Discussion}

We have demonstrated different proof-of-principle opto-electronic modules with the integration of alignment structures, allowing for laser-assisted adjustment. The alignment range is about $10-20 \mu \mathrm{m}$ with an accuracy of $0.1 \mu \mathrm{m}$. This is sufficient to compensate for PWS and PRS.

Most parts are manufactured using conventional methods, such as milling and drilling. The tuning frames are fabricated using electrical discharge machining. The total fabrication costs are mainly determined by the labor costs of manufacturing the specific handmade subcomponents and the assembly process of the modules. We continue this approach and foresee a major reduction of costs in further simplified one-piece module designs, which can be manufactured with punch and fold techniques. Also, automated feedback in combination with the laser adjustment mechanism moves this technology towards more advanced techniques for semimass or mass production.

\section{Acknowledgments}

This work was supported by the Netherlands Organization for Scientific Research under the "NRC photonics" Grant.

\section{References}

1. Lin, Y. et al, "WIAD Minimization in Butterfly Laser Module Packages: Clip Design," IEEE Trans. On Adv Packag. Vol. 30, No. 3 (2007), pp. 499 - 505.

2. Masubuchi, K. Analysis of welded structures, Pergamon Press Ltd, (Oxford, 1980).

3. Goldak, J. et al, "A New Finite Element Method for Welding Heat Sources," Metallurgical Transactions B. Vol. 15B (1984), pp. 299-305.

4 “MSC.MARC User's Guide," Vol. E, MSC.Software Corporation, 2007

5. Tsirkas, S.A. et al, "Numerical Simulation of Laser Welding Process in Butt-joint Specimens," J. Mater. Process. Technol. 134 (2003) pp. 59-69.

6. Anantathanasarn, S. et al, "Lasing of Wavelengthtunable (1.55 $\mu \mathrm{m}$ region) InGaAsP/InP (100) Quantum Dots Grown by Metal Organic Vapor-phase 
Epitaxy," Applied Physics Letters. Vol. 89, No 7 (2006), pp. 073115-1/3.

7. Zantvoort, J.H.C. van. et al, "Effective Pigtailing Method for Fiber Arrays to InP-based Photonic Integrated Circuits" IEEE Trans. On Adv. Packag. (4/2008 Accepted).

8. Zantvoort, J.H.C. van. et al, "Lensed Fiber Array Assembly with Individual Fiber Fine Positioning in the Submicrometer Range" IEEE J. Selec. Topics Quantum Electron, Vol. 12, No. 5 (2006), pp. 931939. 
